\title{
Effect of sex difference on the histological structure of bronchus and nasal cavity in animal asthma model
}

\author{
Reinaldi Rachmadhianto ${ }^{1}$, Tri Hartini Yuliawati ${ }^{*}$ (D) Gatot Soegiarto ${ }^{3}$ \\ ${ }^{1}$ Medical Program, Faculty of Medicine, Universitas Airlangga, Surabaya, Indonesia. ${ }^{2}$ Department of \\ Anatomy, Histology and Pharmacology, Faculty of Medicine, Universitas Airlangga, Surabaya, Indonesia. \\ ${ }^{3}$ Division of Allergy \& Clinical Immunology, Department of Internal Medicine, Dr. Soetomo General \\ Academic Hospital, Surabaya, Indonesia.
}

\begin{tabular}{l}
\hline Article Info \\
\hline Article history: \\
Received Oct, 152019 \\
Revised Dec 1, 2019 \\
Accepted Dec 9, 2019 \\
Published Jan 1, 2020 \\
\hline
\end{tabular}

Keywords:

Asthma

Sex difference

Respiration

Airway

Disease

Chronic respiratory

disease

\begin{abstract}
Background: The prevalence of asthma in prepuberty women is more common than in puberty men. Even after puberty, to a certain age, women dominate more. It is still unclear whether sex hormones affect the histological structure of male and female airways. Objective: This study objective was to examine the effect of sex difference on the histological structure of bronchial and nasal cavity of mice model (Mus musculus) with ovalbumin exposure. Materials and Methods: This study used 24 mice in four groups (male control, female control, male asthma model, and female asthma model). At the sensitization phase in days 0 and 14 , the mice were injected intraperitoneally with $100 \mu \mathrm{l}$ of a mixture of $50 \mu \mathrm{l}$ ovalbumin (200 $\mu \mathrm{l} / \mathrm{ml}$ ) and $50 \mu \mathrm{l}$ alum. At the exposure phase in days 21 to 23 , the mice were exposed to $1 \%$ ovalbumin (aerosol, 30 minutes/per day). The mice were sacrificed 48 hours after the last exposure. The data taken included four variables, bronchial epithelial thickness, bronchial smooth muscle thickness, nasal cavity goblet cells number, and nasal cavity mucosal thickness. Then, Shapiro-Wilk normality test and parametric t-test were conducted. Results: In animal asthma models, mice with male and female exposure did not cause differences in epithelial thickness and smooth muscle thickness in bronchus compared to the control group. However, there were significant differences in the number of goblet cells and mucosal thickness in nasal cavity of male and female mice compared to the control group (respectively, $p=0.002 ; p=0.006$ and $p=0.003$; $\mathrm{p}=0.005$ ). There were no significant differences between groups of male and female mice on all variables. Conclusion: In animal asthma models of mice, ovalbumin exposure did not cause differences in the values of all variables between male and female groups.
\end{abstract}

\section{Corresponding Author:}

Tri Hartini Yuliawati

Department of Anatomy, Histology and Pharmacology, Faculty of Medicine, Universitas Airlangga Jl. Mayjen. Prof. Moestopo no. 47, Surabaya 60131, East Java, Indonesia yulihisto@fk.unair.ac.id

\section{BACKGROUND}

Asthma is a heterogeneous disease that is usually characterized by chronic airway inflammation, a history of respiratory symptoms such as wheezing, shortness of breath, chest tightness and coughing 
whose intensity varies from time to time and accompanied by varying expiratory airflow obstructions (Global Initiative for Asthma, 2018). In general, at the age of children or pre-puberty, asthma attacks more men than women, but after the age of pre-puberty asthma attacks more women (Vink, et al., 2010; Fuseini \& Newcomb, 2017).

Epidemiological data show that adult women suffer more and are more likely to suffer from an asthma exacerbation than men (Zein \& Erzurum, 2015; Fuseini \& Newcomb, 2017). Asthma is one of the most common chronic disease that affect pregnant woman. Exacerbation of asthma during pregnancy is a significant problem and can have a devastating effect. During pregnancy, $45 \%$ of women with asthma experience exacerbations and their rate of hospitalisation was higher (Kim et al, 2015; Murphy, 2015). Women with asthma who experience exacerbations during high-risk pregnancies give birth to babies with the low birth weight with all the associated effects (MacMullen, et al., 2010; Vanders \& Murphy, 2015).

The difference in the incidence of asthma by sex difference cannot be fully explained, but the transition to asthma incidence after puberty implies a hormonal influence. Asthma symptoms in women seem to be influenced by several phases of life, such as puberty, menstruation, pregnancy, and menopause (Shah \& Newcomb, 2018). Airway sensitivity in asthma patients is usually measured by the provocative concentration of methacholine needed to cause a 20\% decrease in FEV1 from basal (PC20). Adult women with controlled asthma experience a decrease in PC20 of more than half during the menstrual cycle, the lowest PC20 value occurs at peak concentrations of estrogen and progesterone in the luteal phase. Therefore, the PC20 change cycle is believed to be influenced by sex-steroid hormones (Matteis et al., 2014; Zein \& Erzurum, 2015; Wei et al., 2015). It is still unclear whether progesterone or estrogen levels or a balance between the two can cause worsening asthma symptoms (Zein \& Erzurum, 2015), but several new studies have shown that endogenous sex hormones can indeed affect lung function and asthma control. Estrogen or estradiol is known to increase Th2-mediated inflammation in the airways after allergen exposure. Estrogen also plays an important role in the secretion of IL-17A, which leads to airway inflammation (Fuseini \& Newcomb, 2017; Yung, et al., 2018).

From this description, it is necessary to prove whether estrogen is indeed a determining factor in the difference of the incidence of asthma by sex difference. Given its effect, estrogen can affect the structure or anatomy of the airways (ie. affect the number of goblet cells and trigger goblet cell metaplasia, which may also contribute to thickening of the airway mucosa) which will ultimately affect the incidence and severity of asthma symptoms in adult women. To prove this, an invasive examination is needed, which is an airway biopsy in female patients with severe asthma. Bronchoalveolar fluid rinses, pulmonary examination and blood tests are also needed. However, invasive examination is certainly constrained technically and ethically. Therefore, research is needed by modeling asthma in experimental animals. Among the several animal models, the asthma model in mice is one of the main choices because mice have a very homologous immune system with humans, the technique is relatively easy and tested, and various commercial reagents are available on the market (Aun et al., 2017; Haspeslagh et al., 2017). We evaluated the histological differences in male and female adult mice that were sensitized and exposed to inhalated allergens, and compared them with control groups that were not treated.

\section{OBJECTIVE}

In general, the objective of this study was to explain the differences in the incidence of asthma in women and men through proving the presence of differences in microscopic structures in the bronchial and nasal cavity of mice (Mus musculus) asthma model with ovalbumin exposure in male and female mice. However, specifically, the objectives of this study were to prove the differences in the thickness of the epithelium and smooth muscle in the bronchus of mice (Mus musculus) asthma models exposed to ovalbumin, and to prove the difference in the number of goblet cells and thickness of mucosa in the nasal cavity of mice (Mus musculus) asthma models exposed to ovalbumin.

\section{MATERIALS AND METHODS}

This research was an experimental study with a post-test only control group design. Inclusion criteria include BALB/c mice (Mus musculus) 6-8 weeks old weighing 20-40 grams (male) and 18-35 grams (female), healthy condition, and active movements. Exclusion was done if there were mice that 
experienced pain that was not caused by the research treatment and were thought to affect the observed variables.

Experimental animals were divided into four groups (male control, female control, male treatment, female treatment). The exposure of ovalbumin was done in two stages, the sensitization, and challenging. In the sensitization stage, the treatment group mice were injected intraperitoneally on days 0 and 14 with $100 \mu \mathrm{l}$ mixture consisting of $50 \mu \mathrm{l}$ ovalbumin $(200 \mu \mathrm{l} / \mathrm{ml})$ and $50 \mu \mathrm{l}$ alum. In the challenging stage, mice were exposed to aerosol $1 \%$ ovalbumin for three days, starting from day 21 with the duration of 30 minutes of exposure per day. Then the airway preparation was taken 48 hours after the last exposure.

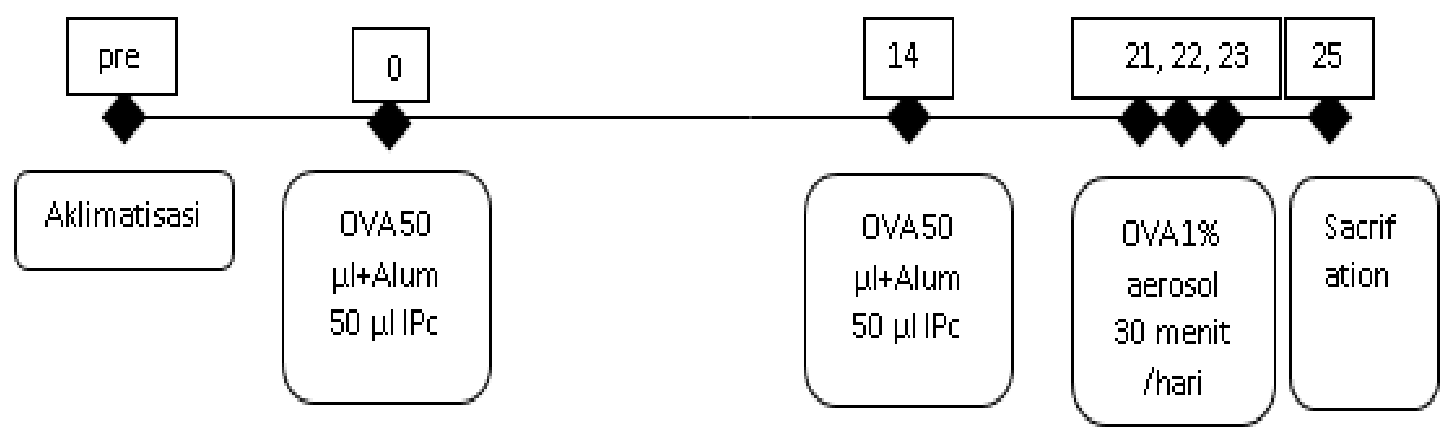

Figure 1. Method's flowchart

The normality of the data distribution was tested using the Shapiro-Wilk test, while different variables were tested using the independent sample t-test. Data were analyzed using legal SPSS software. This study was approved by the Ethics Committee of the Medical Research Ethics Committee of the Faculty of Medicine, Universitas Airlangga, Surabaya, Indonesia.

\section{RESULTS}

In female mice, the epithelial thickness increased from $18.962 \pm 2.23 \mu \mathrm{m}$ to $20.287 \pm 2.6 \mu \mathrm{m}(\mathrm{p}=$ 0.370). In male mice, smooth muscle thickness increased from $15.219 \pm 4.25 \mu \mathrm{m}$ to $18.596 \pm 2.45 \mu \mathrm{m}$ $(\mathrm{p}=0.152)$. There was no significant difference in the thickness of the bronchial epithelium in male and female mice in the asthma model mice $(\mathrm{p}=0.303)$. The results can be seen in Table 1 , while the histological profile can be seen in Figure 2 and 3.

In female mice, smooth muscle thickness increased from $10.138 \pm 1.75 \mu \mathrm{m}$ to $12.268 \pm 4.19 \mu \mathrm{m}$ (p $=0.277$ ). In male mice, an increase in the thickness of smooth muscle occurred from $7.048 \pm 1.85 \mu \mathrm{m}$ to $11.142 \pm 2.18 \mu \mathrm{m}(\mathrm{p}=0.015)$. There was no significant difference in bronchial smooth muscle thickness in male and female mice in the asthma model mice $(\mathrm{p}=0.602)$. The results can be seen in Table 1, while the histological profile can be seen in Figure 2 and Figure 3. In female mice, the number of goblet cells increased from $2.167 \pm 1.59$ to $5.833 \pm 1.51(\mathrm{p}=0.006)$. In male mice, an increase in the number of goblet cells from $1.958 \pm 1.44$ to $4.583 \pm 1.18(\mathrm{p}=0.002)$. There was no significant difference in the number of nasal cavity goblet cells between male and female mice in the asthma model mice ( $\mathrm{p}$ $=0.140$ ). The results can be seen in Table 2, while the histological profile can be seen in Figure 4 and Figure 5.

In female mice, the thickness of the mucosa increased from $26.502 \pm 1.68 \mu \mathrm{m}$ to $43.511 \pm 9.01 \mu \mathrm{m}$ $(\mathrm{p}=0.005)$. In male mice, the mucosal thickness significantly increased from $27.147 \pm 3.55 \mu \mathrm{m}$ to $40.479 \pm 7.83 \mu \mathrm{m}(\mathrm{p}=0.003)$. There was no significant difference in mucosal thickness of the nasal cavity between male and female mice in the asthma model mice $(\mathrm{p}=0.548)$. The results can be seen in table 2, while the histological profile can be seen in Figure 4 and Figure 5. 
Table 1. Epithelial layer and smooth muscle layer thickness of the bronchus

\begin{tabular}{lcc}
\hline & Epithelial thickness $(\mu \mathrm{m})$ & Smooth muscle thickness $(\mu \mathrm{m})$ \\
\hline Male Asthma Model & $15.219 \pm 4.25$ & $7.505 \pm 1.85$ \\
$\quad$ Control & $18.596 \pm 2.45$ & $11.142 \pm 2.18^{*}$ \\
$\quad \begin{array}{l}\text { Treatment } \\
\text { Female Asthma Model }\end{array}$ & $18.962 \pm 2.23$ & $10.138 \pm 1.75$ \\
$\quad \begin{array}{l}\text { Control } \\
\quad \text { Treatment }\end{array}$ & $20.287 \pm 2.64$ & $12.268 \pm 4.19$ \\
Comparation of Asthma Model & $18.596 \pm 2.45$ & $11.142 \pm 2.18$ \\
$\quad$ Male Treatment & $20.287 \pm 2.64$ & $12.268 \pm 4.19$ \\
$\quad$ Female Treatment & $\mathrm{p}<0.05=$ significant value $\mathrm{p}>0.05=$ insignificant value
\end{tabular}

Table 2. Goblet cells number and mucosal layer thickness on nasal cavity

\begin{tabular}{lll}
\hline & Goblet Cells Number $($ per $200 \mu \mathrm{m})$ & Mucosal Thickness $(\mu \mathrm{m})$ \\
\hline Male Asthma Model & $1.958 \pm 1.44$ & \\
$\quad$ Control & $4.583 \pm 1.18$ & $27.147 \pm 3.55$ \\
$\quad$ Treatment & $2.167 \pm 1.59$ & $40.479 \pm 7.83$ \\
$\begin{array}{l}\text { Female Asthma Model } \\
\quad \text { Control }\end{array}$ & $5.833 \pm 1.51$ & $26.502 \pm 1.68$ \\
$\quad \begin{array}{l}\text { Treatment } \\
\text { Comparation of Asthma Model }\end{array}$ & $4.583 \pm 1.18$ & $43.511 \pm 9.01$ \\
$\quad$ Male Treatment & $5.833 \pm 1.51$ & $40.479 \pm 7.83$ \\
$\quad$ Female Treatment & $\mathrm{p}<0.05=$ significant value $\mathrm{p}>0.05=$ insignificant value
\end{tabular}

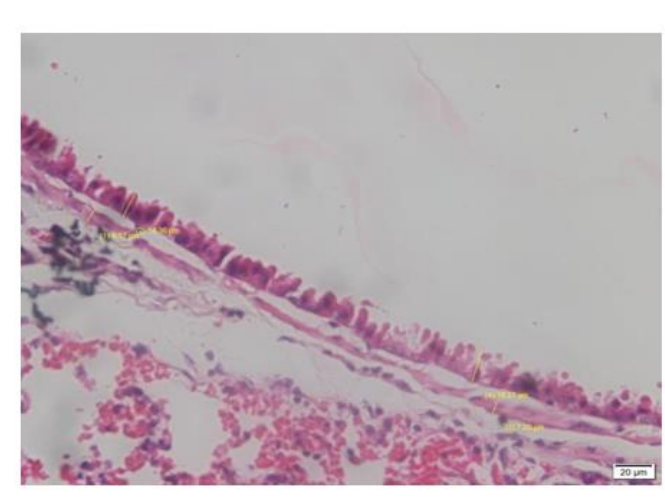

Figure 2. Bronchial histology of male mice with HE staining

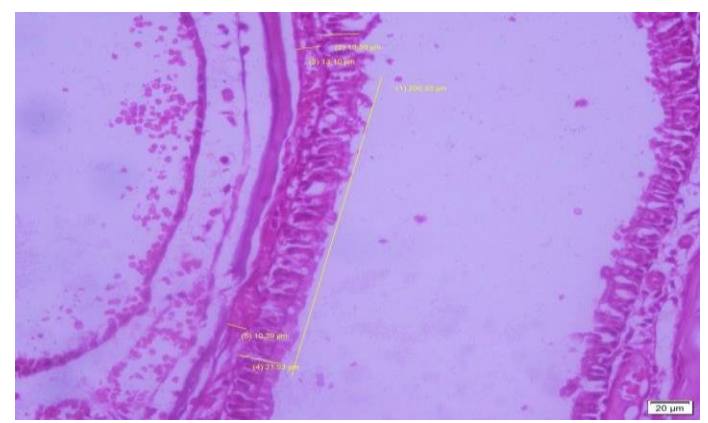

Figure 4. Nasal cavity histology of male mice with HE staining

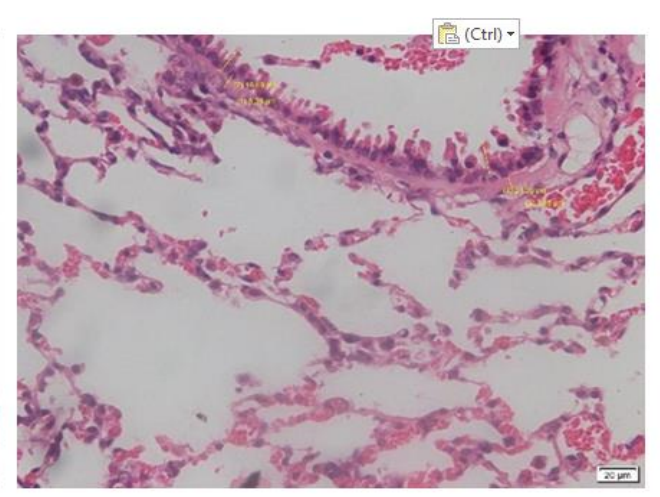

Figure 3. Bronchial histology of female mice with HE staining

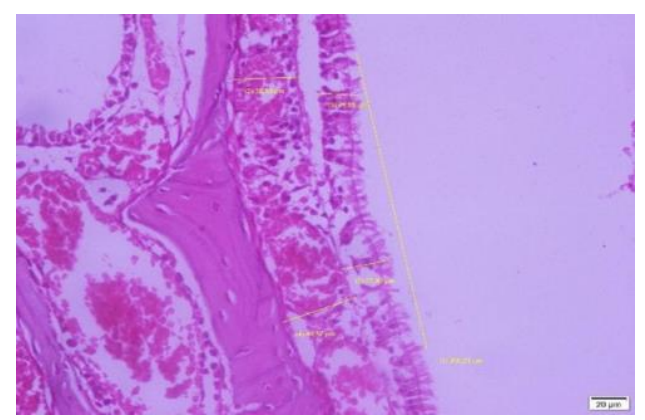

Figure 5. Nasal cavity histology of female mice with HE staining

\section{DISCUSSION}

Chronic Th2 type inflammation in the airways can cause structural changes in the airway mucosa and activation of various immune cells, which will eventually cause deterioration of the lung physiology 
over time. Most immune cells express estrogen receptors (ER $\alpha$, ER $\beta$, or membrane-bound G-proteincoupled ER) and can, therefore, respond to these hormones. Through its interaction with these receptors, estrogen can regulate the differentiation and activation of several immune cells (macrophages, dendritic cells, eosinophils, mast cells, airway epithelial cells, T lymphocytes and B lymphocytes) including their migration processes, survival, cytokine production and antibodies (Keselman \& Heller, 2015). Through Th2 type inflammation, estrogen indirectly plays a role in goblet cell metaplasia in the airways (Boucherat, et al., 2013). In addition, estradiol supplementation was also able to increase the number of goblet cells and trigger mucous synthesis in the airway epithelium (Tam, et al., 2014). Thus, estrogen is reasonably suspected as a determinant of the difference in the incidence of asthma in women and men after puberty.

\section{Effect of sex difference on the thickness of the bronchial epithelial layer}

Before the treatment of asthma modeling, there were differences in the thickness of the bronchial epithelial layer between male mice and female mice. However, this difference was not statistically significant. In female mice, the thickness of the bronchial epithelial layer was $18.9623 \pm 2.23 \mu \mathrm{m}$, whereas, in male mice, the thickness was $15.2190 \pm 4.25 \mu \mathrm{m}$. Modeling asthma with ovalbumin exposure in mice was proven to have caused changes in the thickness of the bronchial epithelial layer in both male and female mice, but these were also not statistically significant (respectively $p=0.370$ and $p=0.152$ ). This can be caused by the short period of ovalbumin exposure in the animal asthma modeling procedure chosen by the researcher.

The procedure of modeling asthma in experimental animals that the researchers used included a brief treatment with a total of 25 days and ovalbumin exposure for only three days, in contrast to the asthma modeling conducted by Li et al. (2013) with a total treatment for 52 and 43 days and with ovalbumin exposure for 14 days (Li, et al., 2013). The relatively short exposure time in this study was able to cause an increase in the thickness of the epithelial layer of mice in both male and female asthma models but did not reach the statistical significance limit.

Meanwhile, there was no difference in the thickness of the bronchial epithelial layer between male and female mice in both the control mice group $(\mathrm{p}=0.085)$ and the asthma model mice group $(\mathrm{p}=$ 0.303). This is in line with Blacquière et al.'s research, which states that sex difference (male or female) does not cause structural differences in the airways of experimental animals. However, significant differences are found in several other variables, such as eosinophil counts and cytokine levels (Blacquière, et al., 2010).

\section{Effect of sex difference on the thickness of the bronchial smooth muscle layer}

Before the treatment of asthma modeling, there were differences in the thickness of the bronchial epithelial layer between male mice and female mice. Supposedly, the difference is also not statistically significant, but this research showed significant results. In female mice, the thickness of the bronchial smooth muscle layer was $10.1379 \pm 1.75 \mu \mathrm{m}$, whereas, in male mice, the thickness was $7.508 \pm 1.85$ $\mu \mathrm{m}$. Modeling asthma with ovalbumin exposure in mice had shown to cause changes in the thickness of the bronchial smooth muscle layer in both male and female mice. This was not statistically significant in female mice but was statistically significant in male mice ( $\mathrm{p}=0.277$ and $\mathrm{p}=0.015$, respectively). This can be caused due to the reasons previously explained.

Meanwhile, there was difference in thickness of the smooth muscle layer between male and female mice in the control group $(p=0.030)$, but not in the asthma model mice group $(p=0.602)$. This is in line with Blacquière et al.'s research, which states that sex difference (male or female) does not cause structural differences in the airways of experimental animals. However, significant differences are found in several other variables, such as eosinophil counts and cytokine levels (Blacquière, et al., 2010).

\section{Effect of sex difference on the nasal cavity's goblet cells number}

Before the treatment of asthma modeling, there had been differences in the number of nasal cavity's goblet cells between male mice and female mice. However, this difference was not statistically significant. In female mice, the number of nasal cavity's goblet cells (per $200 \mu \mathrm{m}$ ) was $2.1667 \pm 1.59$, while in male mice, the number was $1.9583 \pm 1.44$. Modeling asthma with ovalbumin exposure in mice was proven to have caused changes in the number of nasal cavity's goblet cells in both male and female mice, it was also statistically significant (respectively $\mathrm{p}=0.006$ and $\mathrm{p}=0.002$ ). This is in line with research by Zhang et al. (2019) related to synovial inflammation with methods similar to this study 
(using ovalbumin as exposure material) in which images of goblet cell hyperplasia are obtained with a value of significant (Zhang, et al., 2019).

Then, interestingly, in animal asthma modeling, histological structure changes (in this case is a significant difference in the number of goblet cells) were seen in the nasal cavity. The researchers' findings that the modeling of experimental animal asthma also caused a significant increase in the number of nasal cavity's goblet cells, which could be viewed as findings that support the theory of United Airway Disease. From an anatomical aspect, the nasal cavity is located in the upper airway while the bronchi are in the lower airway. Although both have anatomical and histological similarities such as the epithelium, lamina propria, and goblet cells, they are both very far apart. In research using animal models of this asthma, an inflammatory model was formed in the nasal cavity. This is in accordance with the theory of United Airway Disease or One Way, which states that the airway is a unity, and the inflammatory process along it has a very similar etiopathogenic mechanism. Epidemiologically, clinically, and pathophysiologically, there is a strong relationship between airway diseases, especially asthma and rhinosinusitis (Labò, et al., 2011; Licari, et al., 2014; Giavina-Bianchi, et al., 2016).

Meanwhile, there were no differences in the number of goblet cells between male and female mice in the control mice group $(p=0.816)$ and the asthma model group $(p=0.140)$. This suggests that the sex (male or female) does not cause structural differences in the airways of experimental animals (in this case, the number of goblet cells in the nasal cavity).

\section{Effect of sex difference on the thickness of the nasal cavity's mucosal layer}

Before the treatment of asthma modeling, there were differences in the thickness of the mucous layer of nasal cavity between male mice and female mice. However, this difference was not statistically significant. In female mice, the thickness of the nasal cavity mucosa was $26.5022 \pm 1.68 \mu \mathrm{m}$, whereas, in male mice, the thickness of the mucosa was $27.1473 \pm 3.55 \mu \mathrm{m}$. Modeling asthma with ovalbumin exposure in mice has been shown to cause an increase in the thickness of the mucous layer of nasal cavity in both male and female mice, it was also statistically significant $(0.005 *$ and $0.003 *$ respectively). This is in line with Mendiola et al.'s (2016) study related to sino-nasal inflammation in ovalbumin-exposed mice, although the method used is slightly different. However, this study was also in line with research by Kim et al. (2017) where the nasal cavity epithelial hyperplasia (which of course also affects the mucosa) in mice with asthma models was exposed to proteases and ovalbumin (Mendiola, et al., 2016; Kim, et al., 2017).

Then, it was an interest that in animal asthma modeling, changes in histological structure (in this case, the thickness of the mucous layer of nasal cavity) were seen in the nasal cavity. These results support the theory of the United States Airway Disease with the same explanation as previously reviewed.

Meanwhile, there were no differences in the number of goblet cells between male and female mice in the control mice group ( $\mathrm{p}=0.699)$ and the asthma model group ( $\mathrm{p}=0.548)$. This suggests that the sex (male or female) does not cause structural differences in the airways of experimental animals (in this case, the thickness of the mucous layer in the nasal cavity).

\section{CONCLUSION}

Experimental studies using mice (Mus musculus) which were exposed to ovalbumin as asthma models using 24 mice in four groups (male control, female control, male treatment, and female treatment) as well as four variables (thickness of bronchial epithelium, bronchial smooth muscle thickness, thickness of bronchial smooth muscle, thickness of male nasal cavity's mucosa, and the number of nasal cavity's goblet cells) indicates that sex difference (male or female) does not cause differences in the thickness of the epithelium and smooth muscle in the bronchus of mice (Mus musculus) asthma models, which is exposed to ovalbumin. Sex difference (male or female) does not cause a significant difference in the number of goblet cells and thickness of mucosal in the nasal cavity of mice (Mus musculus) asthma models, which is exposed to ovalbumin. Animal asthma modeling with ovalbumin exposure is able to cause an increase in the thickness of the bronchial mucous layer, thickness of the bronchial smooth muscle layer, the number of nasal cavity's goblet cells, and the thickness of the nasal cavity's mucous layer, both in male mice (Mus musculus) female, but sex difference (male or female) does not cause significant differences in the microscopic structure of the 
bronchi and the nasal cavity. Thus, it is suspected that other factors are more dominant (other than sex factors) that can explain the difference in the incidence of asthma in women and men, which still requires further research.

\section{REFERENCES}

Aun, M. Bonamichi-Santos, R. Arantes- Costa, F. M. Kalil, J. Giavina-Bianchi, P., 2017. Animal models of asthma: utility and limitations. Journal of Asthma and Allergy, 10: 293-301.

Blacquière, M., Hylkema, M., Postma, D., Geerlings, M., Timens, W., Melgert, B., 2010. Airway inflammation and remodeling in two mouse models of asthma: Comparison of males and females. International Archives of Allergy and Immunology, 153(2): 173-81.

Boucherat, O., Boczkowski, J., Jeannotte, L., Delacourt, C., 2013. Cellular and molecular mechanisms of goblet cell metaplasia in the respiratory airways. Experimental Lung Research, 207-16.

Fuseini, H., Newcomb, D.C., 2017. Mechanisms driving gender differences in asthma. Current Allergy and Asthma Reports.

Giavina-Bianchi, P., Aun, M., Takejima, P., Kalil, J., Agondi, R., 2016. United airway disease: current perspectives. Journal of Asthma and Allergy, 93.

Global Initiative for Asthma, 2018. Global strategy for asthma management and prevention. Dowloaded from http://ginasthma.org

Haspeslagh, E., Debeuf, N., Hammad, H., Lambrecht, B., 2017. Murine models of allergic asthma. Methods in Molecular Biology, 121-136.

Keselman, A., Heller, N., 2015. Estrogen signaling modulates allergic inflammation and contributes to sex differences in asthma. Frontiers in Immunology.

Kim, S., Kim, J., Park, S.Y., Um, H.Y., Kim, K., Kim, Y., Park, Y., Baek, S., Yoon, S.Y., Kwon, H.S., Cho, Y.S., Moon, H.B., Kim, T.B., 2015. Effect of pregnancy in asthma on health care use and perinatal outcomes. J Allergy Clin Immunol, 136(5): 1215-23.e1-6.

Kim, H., Lim, J., Kim, S., Kim, J., Jang, Y., 2017. Development of a mouse model of eosinophilic chronic rhinosinusitis with nasal polyp by nasal instillation of an Aspergillus protease and ovalbumin. European Archives of Oto-Rhino-Laryngology, 274(11): 3899-3906.

Labò, A., Marseglia, E., Ciprandi, A., La Rosa, G.M., 2011. Nasal disease and asthma. International Journal of Immunopathology and Pharmacology, 24: 7-12.

Li, B., Luo, Q., Nurrahmat, M., Jin, H., Du, Y., Wu, X., Lv, Y., Sun, J., Abduwaki, M., Gong, W., Dong, J. 2013. Establishment and comparison of combining disease \& syndrome model of asthma with 'kidney yang deficiency' and 'abnormal savda'. Evidence-Based Complementary and Alternative Medicine, 2013. Downloaded from http://dx.doi.org/10.1155/2013/658364.

Licari, A., Caimmi, S., Bosa, L., Marseglia, A., Marseglia, G., Caimmi, D. 2014. Rhinosinusitis and Asthma: A very long engagement. International Journal of Immunopathology and Pharmacology, 27(4): 499-508.

MacMullen, N., Shen, J. Tymkow, C., 2010. Adverse maternal outcomes in women with asthma versus women without asthma. Applied Nursing Research, 23(1).

Matteis, M., Polverino, F., Spaziano, G. Roviezzo, F., Santoriello, C., Sullo, N., Bucci, M.R., Rossi, F., Polverino, M., Owen, C.A., D'Agustino, B. 2014. Effects of sex hormones on bronchial reactivity during the menstrual cycle. BMC Pulm Med ,14: 108.

Mendiola, M., Tharakan, A., Chen, M., Asempa, T., Lane, A. Ramanathan, M., 2016. Characterization of a novel high-dose ovalbumin-induced murine model of allergic sinonasal inflammation. International Forum of Allergy and Rhinology, 6(9): 964-72.

Murphy, V., 2015. Managing asthma in pregnancy. Breathe, 11(4): 258-67.

Shah, R., Newcomb, D.C., 2018. Sex bias in asthma prevalence and pathogenesis. Frontiers in Immunology, 9.

Tam, A., Wadsworth, S., Dorscheid, D., Man, S., Sin, D., 2014. Estradiol increases mucus synthesis in bronchial epithelial cells. PLoS ONE, 9(6).

Vanders, R.L., Murphy, V.E., 2015. Maternal complications and the management of asthma in pregnancy. Women's Health, 183-91. 
Vink, N., Postma, D, Schouten, J., Rosmalen, J., Boezen, H., 2010. Gender differences in asthma development and remission during transition through puberty: The TRacking Adolescents' Individual Lives Survey (TRAILS) study. Journal of Allergy and Clinical Immunology, 126(3).

Wei, J., Gerlich, J., Genuneit, J., Nowak, D., Vogelberg, C., Von Mutius, E., Radon, K., 2015. Hormonal factors and incident asthma and allergic rhinitis during puberty in girls', Annals of Allergy, Asthma and Immunology, 115(1): 21-7.

Yung, J., Fuseini, H. Newcomb, D., 2018. Hormones, sex, and asthma. Annals of Allergy, Asthma and Immunology, 488-94.

Zein, J.G., Erzurum, S.C., 2015. Asthma is different in women. Current Allergy and Asthma Reports.

Zhang, Y., Shin, H., Lee, J., Lee, J., 2019. Antiallergic effect of hizikia fusiformis in an ovalbumininduced allergic rhinitis mouse model. Clinical and Experimental Otorhinolaryngology, 12(2): 196205. 